\title{
Epidemiology and Digestive Disease
}

In Paris 1937, just 21 years ago, the International Congress of Gastro-Enterology discussed the Early Diagnosis of Gastric Cancer, and over 700 pages of the Proceedings of the Congress were devoted to that subject. In looking back on these twenty-one years and reviewing achievements during this time, gastric cancer may perhaps serve as an interesting example, for in 1956 at the Congress in London, Pre-Malignant conditions of the stomach and the early diagnosis of gastric cancer was again discussed; again emphasis was laid upon predisposing causes rather than upon diagnosis and treatment. Gastro-Enterology - and indeed Internal Medicine as a whole - has tended in recent years to separate perhaps too much into three main channels, Clinical Medicine, Scientific Medicine und Social Medicine, though they should not in fact develop separately at all.

In gastric carcinoma scientific medicine has made no great advance, but there have been suggestive indications from the laboratory that blood groups bear some genetic relationship to it. It was J7/8. Aird (1953) and others at the Post-Graduate School in London, who first recorded the remarkable association of Gastric Cancer with the blood group A substance. Later studies in England by Jennings and others (1956) have suggested that the excess of group A patients was greatest in cases of pre-pyloric cancer, so that the susceptibility to cancer in individuals possessing the A blood-group substance is not only related to the cancer itself but to the situation in the stomach in

364

\section{Editorial}

which it occurs. The significance of these laboratory and epidemiolo-gical studies is certainly of importance, though the mechanisms concerned are not yet fully known.

Clinically the diagnosis of gastric cancer still depends upon the judgement and experience of the physician and his history taking, whilst physical examination - as often the case in gastric disorders -remains of little value. Gastroscopy which in 1937 was - if not new - at least a rather little used technique, has made some technical advances though the development of gastric biopsy, improved and more flexible gastroscopes but has not greatly added to its early promise. Social Medicine is but a new name for an old conception, but it is here that we find recent - and perhaps future - advances to be greatest. It is here too that interchange of knowledge at meetings, such as the World Congress in Washington may lead most especially to new ideas. During the last 21 years rapid travel, and progress in the spread of information has given the chance for world medicine to advance, and geographic studies of disease to be properly compared.

Sir A. Hurst, at Guy's Hospital, England, with his enquiring and original mind, was one of the first to make special comparative studies of gastritis and ulcer in Holland and England (1929) and to try to collect evidence that gastritis was a precursor of cancer. His view that achlorhydria was a main predisposing condition to gastric cancer has in recent years been supported by workers from the Mayo Clinic (Comfort, Berkson 1956), whose figures show that the cancer incidence in persons which achlorhydria is approximately eight times higher, than in those with 
low or normal acid secretion. It may prove that Hurst was correct in regarding chronic gastritis as the main cause of gastric cancer.

Social and dietary habits over the world can be now more easily investigated than in his time before the second world war, and already some remarkable differences and promising lines of research have been disclosed. Statistical analysis of occupational and other factors has become more general, more accurate and more uniform, and nutritional surveys and controlled trials more standardised and more comparable.

It is now for example more fully appreciated by doctors that some of the accepted figures for the age incidence of conditions such as peptic ulcer took no account of the numbers of individuals at risk for a particular age period, so that for instance at age 60-70 the per-

Editorial

365

centage of cases of duodenal ulcer arising might in fact be higher in relation to the number of individuals living at that age than the percentage occurring at age 30-40. As the length of life of the nation rises, so will the figures for incidence of disease per decade change also, and it is difficult to fortell how these trends of morbidity and mortality will alter in coming years. During the last 25 years for instance the mortality of men between 55 and 65 in Great Britain has risen to nearly double that of women of the same age, as compared to a figure less than one third higher, in the 1920's, Morris (1957). Gastric cancer, which now shows a mortality at least twice as high in men as in women at all ages, will share in any changes that this trend may take, and it may be that more clues may come in the elucidation of its causes from epideimological and geographical investigations than from any clinical or laboratory research. This is even more likely in the case of peptic ulcer where the real international differences in incidence are only just beginning to be discovered. In Gastro-Enterology as a whole developing study of social class distribution, economic, nutritional, and similar factors in relation to disorders such as ulcerative colitis, gallstones, cirrhosis of the liver, may yield striking results. In cancer of the stomach, the social class distribution is similar to that for gastric - but not for duodenal -ulcer, the number of cases occuring in the lowest social scale being nearly double that in the highest. Not only is it known that the lower income group have this higher rate but it is also clear that certain regions - in Great Britain for instance the rural areas of North Wales - also show an abnormally high gastric cancer incidence.

These fields of enquiry have as yet been only lightly explored and may lead to fresh knowledge, perhaps of dietary factors, of food production or even of soil fertility which at present we can only guest at. The help that medicine may receive from the veterinary surgeons may well increase in coming years, and the possible influence of trace elements in the causation of disease is by no means fully known. A review of this aspect of disease by Payne (1956) provides some provocative ideas on essential minerals in the soil and their relation to diseases in animals. Occupational studies have in the last 21 years given rather disappointing results in the study of Gastro-Enterological problems, but the difficulties are great. In gastric cancer some recent studies in the U.S.A. whilst confirming the remarkable association of this disease with Polish birth - a well accepted genetic relationship - also showed

366

Editorial

a statistically higher stomach cancer rate in men working with blast furnaces, steel works and the like than in other occupations. This seemed to be due to exposure to iron dust and similarly, 
though to a lesser degree, men exposed to sandy dust (silica) as in the building trade had a high gastric cancer rate also.

One group of stomach cancer patients for instance showed 12.5 per cent to have been exposed to iron dust for 12 years or longer as against only 1 per cent of a control group, and in the case of inorganic dust 17.9 per cent as against 7.8 per cent of the controls.

In all these studies one of the most intractable problems is the long period during which the presumed etiological agents must be at work before obvious cancer arises, but it is perhaps this aspect which offers contradictorily the most hopeful prospects in treatment.

Treatment of Gastric Cancer since the days of the Paris Congress of 1937 has shown little really significant change; the results of total gastrectomy - though an operation now much more easily performed than it was - remain disappointing. Diagnosis in spite of progress in radiology, or in the cytology of gastric washings, though it may lead to earlier recognition of some cases, has not been followed by any appreciable reduction in mortality in England or to any marked improvement in prognosis.

In prevention however - during the long pre-malignant period -we may with confidence hope for tangible advances in the future; and if this is true in the unpromising field of Gastric Cancer, is it not much more true in respect to other aspects of Gastro-Enterology ? May not perhaps comparative studies of social and environmental factors in different countries not only bring new knowledge in the causation of gastro-enterological disorders but new friendship amongst doctors and nations in addition.

Bibliography

Kraus, A. S., Levin, M. L. and Gerhardt, T.: Amer. J. publ. Hlth 47: 961 (1957. -Payne, E. H.: J. App. Nutrit. 9: 398 (1956). - Aird, I., Bentall, H. and Roberts, J. A.: Brit. Med. J. 1953/1: 799. Jennings, S. D., Balme, R. H. and Richardson, J. E.: Lancet 1956/П: 11. - Comfort, M. W.: Ann. intern. Med. 34: 1331 (1957). -Hurst, A. F.: Lancet 1929/II: 1023. - Berkson, J. B., Comfort, M. W. and Butt, H. R.: Proc. Mayo Clin. 31: 583 (1956). - Morris, J. N.: Uses of Epidemiology.

(Livingstone, Edinburgh 1957.)

$\mathrm{TL} \cdot \mathrm{H}$. 